Co-sputtered MoRe thin films for carbon nanotube growth-compatible superconducting coplanar resonators

This content has been downloaded from IOPscience. Please scroll down to see the full text.

2016 Nanotechnology 27135202

(http://iopscience.iop.org/0957-4484/27/13/135202)

View the table of contents for this issue, or go to the journal homepage for more

Download details:

IP Address: 132.199.96.74

This content was downloaded on 13/10/2016 at 08:51

Please note that terms and conditions apply.

You may also be interested in:

Fabrication artifacts and parallel loss channels in metamorphic epitaxial aluminum superconducting resonators

C J K Richardson, N P Siwak, J Hackley et al.

Superconducting atomic contacts inductively coupled to a microwave resonator

C Janvier, L Tosi, Ç Ö Girit et al.

Anomalous Temperature Dependence of the Quality Factor in a Superconducting Coplanar Waveguide

Resonator

Zhou Pin-Jia, Wang Yi-Wen and Wei Lian-Fu

High-temperature superconductor thin films

N Klein

Analysis of high quality superconducting resonators: consequences for TLS properties in amorphous

oxides

J Burnett, L Faoro and T Lindström

In situ studies of growth of carbon nanotubes on a local metal microheater

O A Nerushev, J Ek-Weis and E E B Campbell 


\title{
Co-sputtered MoRe thin films for carbon nanotube growth-compatible superconducting coplanar resonators
}

\author{
K J G Götz, S Blien, P L Stiller, O Vavra, T Mayer, T Huber, T N G Meier, \\ $M$ Kronseder, Ch Strunk and A K Hüttel \\ Institute for Experimental and Applied Physics, University of Regensburg, Universitätsstr. 31, D-93053 \\ Regensburg, Germany \\ E-mail: karl.goetz@ur.de and andreas.huettel@ur.de
}

Received 8 October 2015, revised 17 January 2016

Accepted for publication 27 January 2016

Published 22 February 2016

\begin{abstract}
Molybdenum rhenium alloy thin films can exhibit superconductivity up to critical temperatures of $T_{\mathrm{c}}=15 \mathrm{~K}$. At the same time, the films are highly stable in the high-temperature methane/hydrogen atmosphere typically required to grow single wall carbon nanotubes. We characterize molybdenum rhenium alloy films deposited via simultaneous sputtering from two sources, with respect to their composition as function of sputter parameters and their electronic dc as well as $\mathrm{GHz}$ properties at low temperature. Specific emphasis is placed on the effect of the carbon nanotube growth conditions on the film. Superconducting coplanar waveguide resonators are defined lithographically; we demonstrate that the resonators remain functional when undergoing nanotube growth conditions, and characterize their properties as function of temperature. This paves the way for ultra-clean nanotube devices grown in situ onto superconducting coplanar waveguide circuit elements.
\end{abstract}

Keywords: coplanar resonator, carbon nanotube growth, rhenium, molybdenum, XPS, superconductivity

(Some figures may appear in colour only in the online journal)

\section{Introduction}

The discovery of single-walled carbon nanotubes [1, 2] opened the door for a wide range of both fundamental research and technical applications in such electronic and nano-electromechanical systems. These macromolecules exhibit particular properties as, e.g., high mechanical, chemical and thermal stability as well as excellent electronic and thermal conductance. They can carry high currents, display outstanding room temperature transistor characteristics, and may compete in the future with or even replace conventional silicon-based logic devices [3-5]. From a fundamental point of view carbon nanotubes provide a highly promising material for quantum information and computation. The macromolecules act as quasi one-dimensional conductors because of their large aspect ratio; the electrostatic definition of localized potential wells can trap electrons in a nuclear spin free environment with well-defined quantum levels [6-10].
One way to ensure that the carbon nanotubes remain clean and defect-free is to grow them directly on pre-fabricated contact electrodes, such that subsequently no further lithography and/or wet chemistry takes place. This technique has led to a multitude of striking measurement results, from single quantum dot spectroscopy $[9,11]$ to the characterization of high- $Q$ vibrational modes $[12,13]$. Performing highfrequency measurements on single wall carbon nanotube structures is a logical extension. Many techniques applied for such measurements on mesoscopic structures require the use of on-chip radiofrequency electronics, in particular superconducting coplanar waveguides and microwave resonators formed by these $[15,16]$. An example for such a possible combined device structure is sketched in figure 1(a) - a carbon nanotube is capacitively coupled to the coplanar $\lambda / 2$ resonator. In combination with the overgrowth process, this poses extensive difficulties, since the carbon nanotube growth process-typically $10-15 \mathrm{~min}$ in a hot methane-hydrogen 


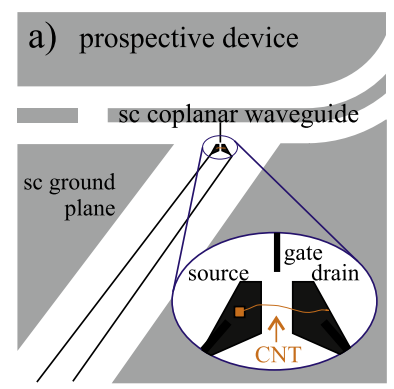

b)

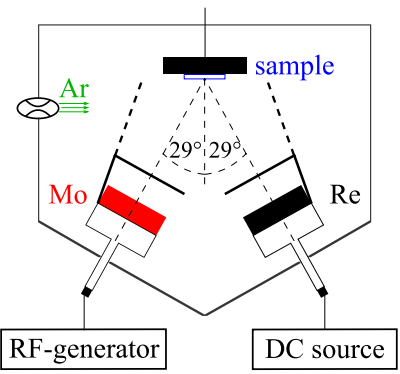

Figure 1. (a) Schematic sketch of a carbon nanotube quantum dot device capacitively coupled to a coplanar $\lambda / 2$ resonator. For similar devices using different material systems, see [14, 15]. (b) Sketch of the Orion dual-source sputtering setup used during fabrication of our metal thin films. The molybdenum target is driven by a radiofrequency source with impedance matching, the rhenium target using a dc source. A mass flow controller sets the argon flow into chamber.

athmosphere [17]—is highly detrimental to superconducting thin films.

Several solutions for this problem have been proposed, including transfer of the nanotube from a growth chip to a second structure $[8,15,16,18,19]$ or capping the superconductor with a protection layer [14]. If the metal film is to survive the CVD conditions, a high melting point, as provided by e.g. rhenium and molybdenum, becomes an important requirement.

Alloys of rhenium and molybdenum have been shown to exhibit superconducting transition temperatures up to $15 \mathrm{~K}$ [20-22]. Rhenium and molybdenum rhenium alloy thin films remain stable under carbon nanotube CVD growth conditions and subsequently still exhibit superconducting behavior [23]. In addition, after in situ growth they provide transparent electronic contacts to carbon nanotubes [9, 23, 24].

In the following, we characterize molybdenum rhenium alloy films deposited via simultaneous sputtering from two sources, i.e., cosputtering, with respect to their composition as function of sputter parameters and their $\mathrm{dc}$ as well as $\mathrm{GHz}$ properties at low temperature. Specific emphasis is placed on the effect of the carbon nanotube growth conditions on the film. Coplanar waveguide $\lambda / 4$ resonators are defined lithographically and characterized at dilution refrigerator temperatures; even after undergoing the growth conditions internal quality factors of up to $Q_{\mathrm{i}} \simeq 5000$ can be found. The temperature dependence of the resonance frequency and the internal quality factor is evaluated and found consistent with theoretical models.

\section{Thin film deposition}

Figure 1(b) sketches the setup used for the film fabrication. The UHV-chamber with typical pressures in the range of $10^{-8}$ to $10^{-7} \mathrm{mbar}$ contains two sputter targets. The sample holder is placed approximately $13.5 \mathrm{~cm}$ above and in the middle of both targets which are mounted at a distance of circa $15 \mathrm{~cm}$ to each other. Argon gas is injected via a mass flow controller close to the molybdenum target, and a plasma is ignited using a radiofrequency drive at a chamber pressure of $10^{-1} \mathrm{mbar}$.

Subsequently the chamber pressure is reduced to $7 \cdot 10^{-3}$ mbar and the argon plasma at the rhenium electrode is ignited using a dc power supply; at the same time the rfoutput is adjusted such that the plasma close to the molybdenum electrode and target remains stable. As soon as both targets are sputtered, the shutters are opened and the deposition of the MoRe alloy starts. By keeping the chamber pressure constant over the whole process time, the deposition rate of both materials is kept approximately constant. In the following the alloy composition of the films is varied only by tuning one parameter, namely the output power $P_{\text {Mo }}$ of the rfgenerator at the molybdenum target.

\section{Resulting alloy}

In order to obtain the alloy composition, $\mathrm{x}$-ray photoelectron spectroscopy (XPS) is performed on the cosputtered films $[25,26]$. Within a predefined area of four to ten square millimeters the samples are irradiated by a monochromatic x-ray source, and the resulting emitted photoelectrons are collected and spectroscopically analyzed with respect to energy and intensity. The chemical sensitivity is given by the elementspecific distribution of binding energies $E_{\mathrm{B}}$ of the electronic core levels.

Characteristic XPS data of a cosputtered film are plotted in figure 2(a). The signal peaks corresponding to atomic and molecular core levels have been identified following [27]. The black curve in figure 2(a) reveals the chemical composition of the topmost surface layers. This is due to the small mean free path of the photoexcited electrons within the film material of few nanometers. In addition to characteristic peaks originating from molybdenum and rhenium core levels, also significant oxygen and carbon peaks are observed. This is likely due to the fact that all samples have been exposed to air during transfer from the sputtering device into the UHV chamber of the XPS setup.

Adsorbates and the film itself can be etched by in situ Arsputtering for obtaining spectra from lower layers. An etching step lasts several minutes at a chamber pressure of less than $3 \cdot 10^{-8} \mathrm{mbar}$ in which the base pressure is of $5 \cdot 10^{-10} \mathrm{mbar}$. Etching steps are repeated until the oxygen 1s-peak only negligibly contributes to the whole spectrum (at most about $10 \%$ ). The red (gray) line in figure 2(a) displays the corresponding spectrum. Note that also the carbon peak is now strongly suppressed. Subsequent tests using a profilometer show that approximately $5 \mathrm{~nm}$ of the films were removed. All spectra obtained after such a corresponding etching step are henceforth denoted as 'bulk' spectra.

Figures 2(b) and (c) display details of the spectrum close to the Mo 3d- and Re 4f-peaks, referenced to the C 1s-peak [26]. The additional structure in the Mo 3d surface spectrum originates from $\mathrm{MoO}_{3}$ and $\mathrm{MoO}_{2}$ forming at the alloy surface $[25,28]$. It decreases subsequently until disappearing in the bulk where only the two Mo $3 \mathrm{~d}_{5 / 2^{-}}$and Mo $3 \mathrm{~d}_{3 / 2}$-peaks are part of the spectrum. In contrast, at the Re $4 \mathrm{f}$-peak neither 

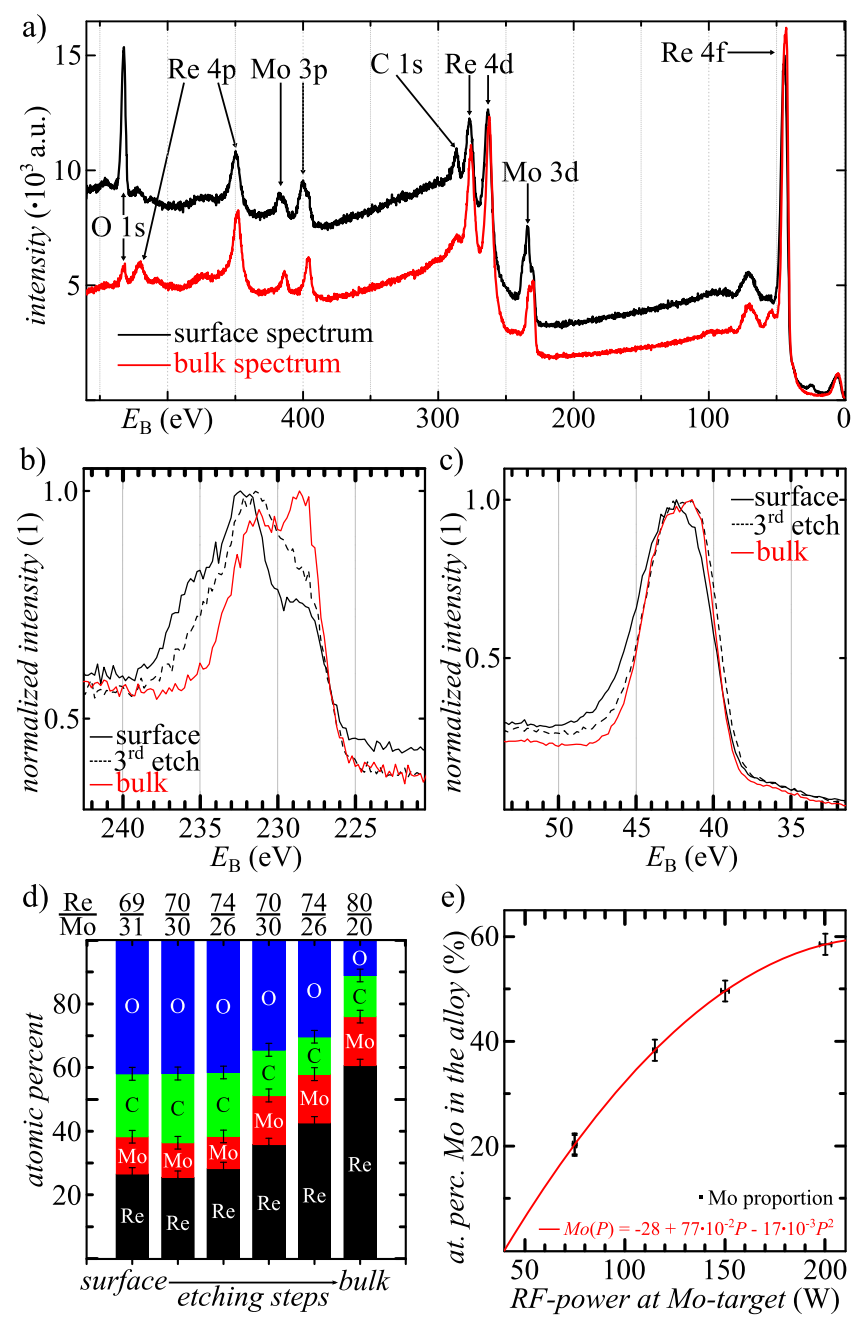

Figure 2. (a) Example x-ray photoelectron spectroscopy (XPS) spectrum of a deposited, unstructured film on a $\mathrm{p}^{++}-\mathrm{Si} / \mathrm{SiO}_{2}$ substrate. A power setting of $P_{\mathrm{Mo}}=75 \mathrm{~W}$ at the molybdenum source was used. The black line represents the chemical composition of the as-grown sample surface, the red line was recorded after removing approximately $5 \mathrm{~nm}$ of the film by in situ argon-ion sputtering beam sputtering within the XPS chamber. Normalized detail spectra close to the Mo 3d-peaks (b) and the Re 4f-peaks (c) (see text). The energetic step size is set to $0.2 \mathrm{eV}$. (d) Depth profile of the atomic composition, calculated from XPS spectra as in (a), from surface to $\sim 5 \mathrm{~nm}$ depth (subsequently named bulk). The resulting MoRe-alloy composition after each step is stated above. (e) Relative molybdenum atomic percentage in the bulk film for different power settings $P_{\text {Mo }}$ at the molybdenum target (see text).

changes in the line shape nor peak shifts are observed in the surface compared to the bulk spectrum, indicating the absence of rhenium oxides at the surface and in the bulk layers. All films examined in this work exhibit the same behavior for the rhenium peaks $[25,26]$.

All rhenium peaks of the film are shifted by $1.3 \mathrm{eV}$ to higher $E_{\mathrm{B}}$-values, compared to the literature values of pure rhenium. Similar values have been identified in [26] as chemical shift due to the Mo-Re compound formation.

Using the method of area sensitivity factors and evaluating the Mo 3d-, Re 4f-, C 1s-, and O 1s-peaks, the atomic concentrations of the sample have been estimated [27]. Figure 2(d) displays the atomic concentrations as a function of depth. While the exposure to air leads to significant carbon and oxygen percentages at the surfaces, these both decrease strongly; for the 'bulk' spectrum about $10 \%$ of carbon remains.

Subsequently the molybdenum-rhenium alloy ratio is obtained by normalizing to the sum of both sputtered metals. The resulting alloy ratios of all co-sputtered films vary as a function of depth, see figure 2(d). This is in agreement with observations in [26] and explained there by diffusion of molybdenum atoms from the bulk to the surface in order to form stable molybdenum oxides, and corresponding enrichment of rhenium atoms in the film interior.

As can be clearly seen in figure 2(e), where the resulting alloy ratio in the bulk film is plotted as a function of $\mathrm{rf}$ power $P_{\text {Mo }}$ applied to the molybdenum sputter source, the resulting molybdenum contribution in the alloy can be controlled over a wide range. The solid line in figure $2(\mathrm{e})$ is a quadratic fit to the data points.

\section{Influence of the nanotube growth environment}

Simulating the carbon nanotube chemical vapor deposition (CVD) growth process typically used to locally grow few clean single-wall carbon nanotubes [17], the chip including the MoRe thin film is heated up in an argon and hydrogen gas flow, then exposed to a methane-hydrogen atmosphere at $850{ }^{\circ} \mathrm{C}$ for several minutes and subsequently cooled down under argon and hydrogen flow.

The XPS of a molybdenum rhenium alloy sputtered with $P_{\mathrm{Mo}}=75 \mathrm{~W}$ after exposure to the CVD environment is shown in figure 3(a), and a normalized detail plot of the Mo 3d peak in figure 3(b). A strong carbon peak is visible even in the bulk. Furthermore in both surface and bulk molybdenum spectra oxide peaks are not observed, as visible in figure 2(b).

Having in mind that molybdenum oxides exhibit drastically lower melting points of $795{ }^{\circ} \mathrm{C}$ for $\mathrm{MoO}_{3}$, and $1100{ }^{\circ} \mathrm{C}$ for $\mathrm{MoO}_{2}$ [29] than pure molybdenum with $2610^{\circ} \mathrm{C}$ [30], the data indicates that reduction of the oxide to atomic molybdenum takes place during CVD.

An additionally possible process is the formation of molybdenum carbides. The expected XPS peak of $\mathrm{Mo}_{2} \mathrm{C}$ $\left(E_{\mathrm{B}}=227.75 \mathrm{eV}\right)$ is very close to that of Mo $3 \mathrm{~d}_{5 / 2}$ $\left(E_{\mathrm{B}}=228 \mathrm{eV}\right)$, which makes detection challenging with our experimental resolution. Interestingly, molybdenum carbides display superconductivity with critical temperatures $6 \mathrm{~K} \lesssim T_{\mathrm{c}} \lesssim 9 \mathrm{~K}$ [31]. However, typically the growth of molybdenum carbide out of pure molybdenum, for example in a nitrogen-xylene atmosphere, takes place at temperatures higher than $850{ }^{\circ} \mathrm{C}$, see [31].

The area sensitivity factor analyzing method results in a bulk composition of $26 \%$ carbon, $22 \%$ molybdenum, and $49 \%$ rhenium plus neglectable oxygen residues. Since the etching time for the bulk spectra has been kept constant, it is obvious that the high carbon contribution does not have its 

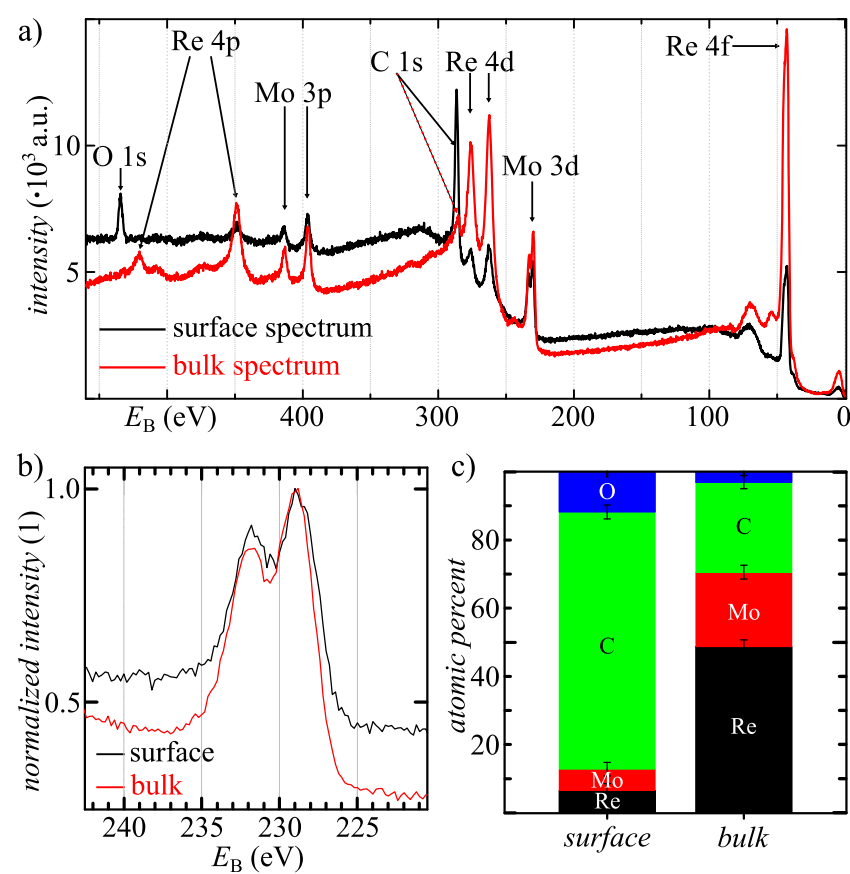

Figure 3. (a) XPS spectrum of a molybdenum rhenium film sputtered at $P_{\mathrm{Mo}}=75 \mathrm{~W}$, after subsequent $30 \mathrm{~min}$ in the carbon nanotube CVD growth environment. The energy step size is set to $0.2 \mathrm{eV}$. (b) Detailed zoom of the Mo 3d peak. (c) Composition of the film at the surface and at $\sim 5 \mathrm{~nm}$ depth (atomic percentage); note the large carbon contribution.

origin in atmospheric adsorbates but in diffusion of carbon into the alloy during CVD. The relative bulk atomic ratio of the sputtered metals is $\mathrm{Mo}_{31} \mathrm{Re}_{69}$, indicating structural changes during CVD. In particular, the high temperature may lead to vertical segregation processes similar to those described in the previous section, but over a larger depth range.

Spectroscopy on a second sample using twice the gas flow of methane results in a composition of $12 \%$ molybdenum, $23 \%$ rhenium, and even $58 \%$ carbon in the bulk. From this we conclude that the penetration of carbon into the alloy also increases. However, the resulting sputtered metal ratio of $\mathrm{Mo}_{34} \operatorname{Re}_{66}$ still remains close to the previous sample.

\section{5. dc characterization}

To characterize the electronic properties of the co-sputtered films at room temperature as well as at cryogenic temperatures, the fabricated thin films on top of $\mathrm{SiO}_{2}$ or $\mathrm{Al}_{2} \mathrm{O}_{3}$ substrates are patterned into Hall bars by means of optical lithography and $\mathrm{SF}_{6} /$ Ar reactive ion etching (see figure 4(a)). Afterwards, selected structures are placed into the CVD furnace and exposed to the nanotube growth environment for several minutes. All measurements have been performed on films either examined by XPS (see last two sections) or deposited simultaneously to these in the same deposition step. Devices using two different alloy compositions have been examined, namely $\mathrm{Mo}_{20} \mathrm{Re}_{80}$ and $\mathrm{Mo}_{58} \mathrm{Re}_{42}$ obtained with $P_{\mathrm{Mo}}=75 \mathrm{~W}$ and $P_{\mathrm{Mo}}=200 \mathrm{~W}$, respectively. a)
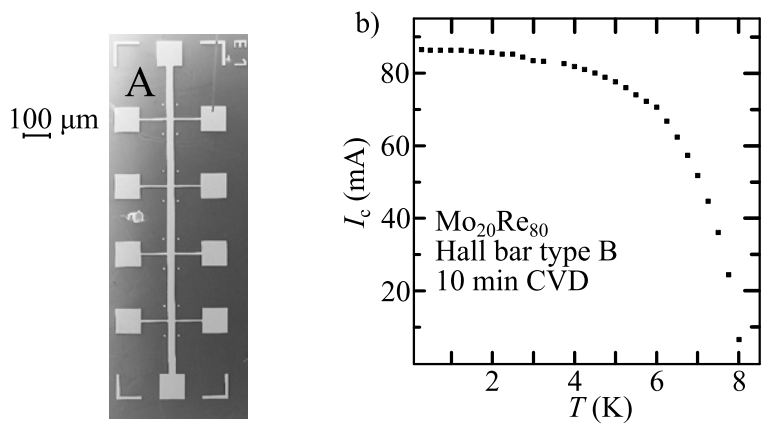

c)
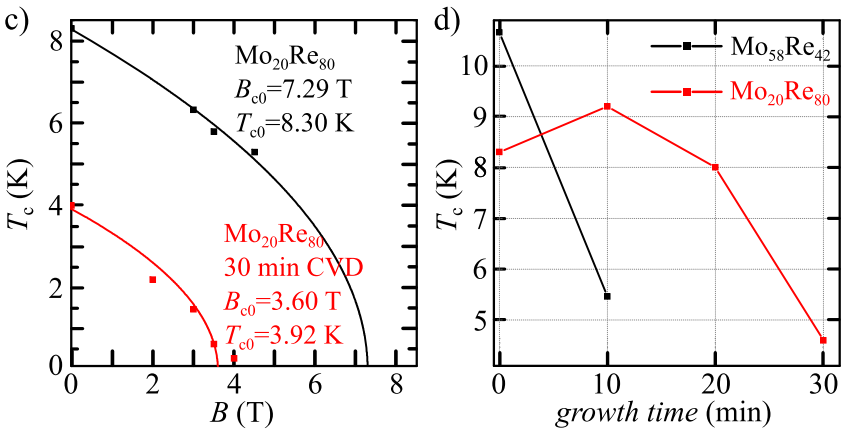

Figure 4. (a) Exemplary SEM micrograph of a test Hall bar structure, type A. Dimensions are for type A width $W_{\mathrm{A}}=28 \mu \mathrm{m}$ and film thickness $d_{\mathrm{A}} \approx 150 \mathrm{~nm}$, for type B $W_{\mathrm{B}}=5 \mu \mathrm{m}$ and $d_{\mathrm{B}} \approx 60 \mathrm{~nm}$. (b) Measured critical current $I_{\mathrm{c}}$ through a Hall bar device (type B),

$\mathrm{Mo}_{20} \mathrm{Re}_{80}$, as a function of temperature. (c) Critical temperature $T_{\mathrm{c}}$ of $\mathrm{Mo}_{20} \mathrm{Re}_{80}$ films as a function of applied magnetic field B, with and without CVD growth exposure. (d) Measured critical temperature $T_{\mathrm{c}}$ as a function of $\mathrm{CH}_{4} / \mathrm{H}_{2}$ flow time during the CVD process. Straight lines are guides to the eye.

At room temperature, compared to $\mathrm{Mo}_{58} \mathrm{Re}_{42}$ both resistivity and sheet resistance of the $\mathrm{Mo}_{20} \mathrm{Re}_{80}$ samples are higher by a factor 3-5: before CVD we obtain $\rho \simeq 3.0 \cdot 10^{-7} \Omega \mathrm{m}$ for $\mathrm{Mo}_{58} \operatorname{Re}_{42}$ and $\rho \simeq 9.0 \cdot 10^{-7} \Omega \mathrm{m}$ for $\mathrm{Mo}_{20} \mathrm{Re}_{80}$. Resistances slightly increase during exposition to the CVD environment, to $\rho \simeq 4.0 \cdot 10^{-7} \Omega \mathrm{m}$ for $\mathrm{Mo}_{58} \mathrm{Re}_{42}$ and $13 \cdot 10^{-7} \Omega \mathrm{m} \lesssim \rho \lesssim 15 \cdot 10^{-7} \Omega \mathrm{m}$ for $\mathrm{Mo}_{20} \operatorname{Re}_{80}$.

Results of low-temperature measurements performed on $\mathrm{Mo}_{20} \mathrm{Re}_{80}$ devices are plotted in figures 4(b) and (c). Independent from CVD-exposure, the residual-rest-resistance values $R R R$ for all $\mathrm{Mo}_{20} \mathrm{Re}_{80}$ devices are in the range $0.8 \lesssim R R R \lesssim 1.0$. Figure 4 (b) displays the critical current of $\mathrm{a} \mathrm{Mo}_{20} \mathrm{Re}_{80}$ film (Hall bar geometry $\mathrm{B}$, with $T_{\mathrm{c}}=9.2 \mathrm{~K}$ ) after $10 \mathrm{~min}$ CVD exposure. It carries a supercurrent up to $I_{\mathrm{c}} \gtrsim 80 \mathrm{~mA}$, corresponding to a critical current density of $j_{\mathrm{c}} \gtrsim 2.7 \cdot 10^{5} \mathrm{~A} \mathrm{~mm}^{-2}$ over a wide temperature range up to ca. $5 \mathrm{~K}$ before the transition to a normal conductor takes place above $8 \mathrm{~K}$. This very high value for $j_{\mathrm{c}}$ is well in accordance with the results of [32], where after high-temperature annealing critical current densities of up to $1.8 \cdot 10^{5} \mathrm{~A} / \mathrm{mm}^{2}$ through suspended $\mathrm{Mo}_{50} \mathrm{Re}_{50}$-nanostructures were reported.

A second $\mathrm{Mo}_{20} \mathrm{Re}_{80}$ Hall bar device (type A, $T_{\mathrm{c}}=8.3 \mathrm{~K}$ ), not exposed to CVD, exhibits a critical current $I_{\mathrm{c}} \approx 114 \mathrm{~mA}$ at $T=4.2 \mathrm{~K}$, corresponding to a lower current density $j_{\mathrm{c}}=2.8 \cdot 10^{4} \mathrm{~A} \mathrm{~mm}^{-2}$. Also much longer exposure to the CVD environment again lowers the reachable critical current density. 


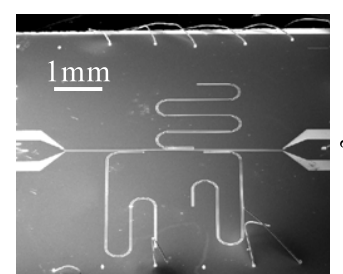

a)
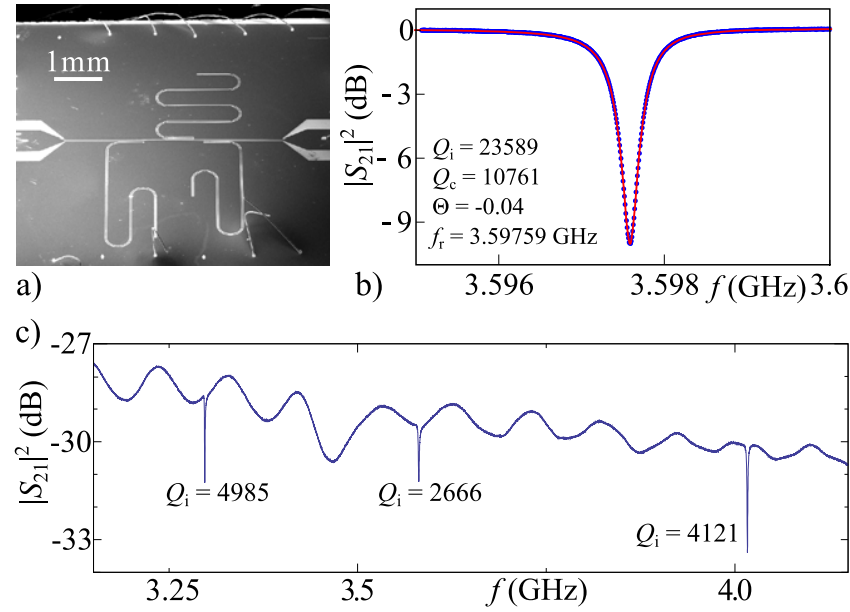

Figure 5. (a) SEM image of a test structure with three MoRe coplanar $\lambda / 4$ resonators coupled to a feed line. (b) Detail of the transmission $\left|S_{21}(f)\right|^{2}$ at $T=100 \mathrm{mK}$ of a device as depicted in (a); the background value of $\left|S_{21}(f)\right|^{2}$ has been subtracted. $150 \mathrm{~nm}$ pristine $\mathrm{Mo}_{20} \mathrm{Re}_{80}$; see the text for a description of the fit. (c) Uncalibrated transmission spectrum $\left|S_{21}(f)\right|^{2}$ at $T=15 \mathrm{mK}$ (dilution refrigerator mixing chamber temperature) of a second $150 \mathrm{~nm}$ $\mathrm{Mo}_{20} \mathrm{Re}_{80}$ device over a wider frequency range, now after $30 \mathrm{~min}$ exposure to the $\mathrm{CVD} \mathrm{CH} / \mathrm{H}_{2}$ flow. Resonances corresponding to the three $\lambda / 4$ structures coupled to the feedline can still be clearly identified.

Figure 4(c) displays data on the magnetic field dependence of the critical temperature. Fitting the empirical relation $B_{\mathrm{c}}(T)=B_{\mathrm{c} 0} \cdot\left(1-\left(T / T_{\mathrm{c} 0}\right)^{2}\right)$ [33] results in high characteristic values $B_{\mathrm{c} 0}=7.3 \mathrm{~T}$ and $T_{\mathrm{c} 0}=8.3 \mathrm{~K}$ expected for molybdenum rhenium alloys, where $T_{\mathrm{c} 0}$ denotes the zero field critical temperature and $B_{\mathrm{c} 0}$ the extrapolated critical field at zero temperature. Again, as observed for critical currents, prolonged (30 min) CVD exposure results in a strong decrease of both values to here $B_{\mathrm{c} 0}=3.6 \mathrm{~T}$ and $T_{\mathrm{c} 0}=3.9 \mathrm{~K}$ $[32,34]$. This effect is also visible in figure $4(\mathrm{~d})$, displaying the critical temperature for two different alloy compositions. Even for only $10 \mathrm{~min}$ growth time, $T_{\mathrm{c}}$ of the $\mathrm{Mo}_{58} \mathrm{Re}_{42}$ film decreases to the half, well in accordance with the results of [34]. Interestingly, in contrast the $\mathrm{Mo}_{20} \mathrm{Re}_{80}$ alloy keeps its critical temperature range of $8 \mathrm{~K} \leqslant T_{\mathrm{c}} \leqslant 9 \mathrm{~K}$ for growth times up to $20 \mathrm{~min}$, only reaching $<5 \mathrm{~K}$ after $30 \mathrm{~min}$ of exposure to the $\mathrm{CH}_{4} / \mathrm{H}_{2}$ flow.

\section{Coplanar resonator devices}

Coplanar waveguide $\lambda / 4$ resonators were fabricated to investigate the high frequency behavior of the alloy material and its suitability for cavity quantum electrodynamics and optomechanics experiments. After sputtering of $150 \mathrm{~nm}$ $\mathrm{Mo}_{20} \mathrm{Re}_{80}$ on $\mathrm{SiO}_{2}$ or $\mathrm{Al}_{2} \mathrm{O}_{3}$ substrates, the structures were patterned using optical lithography and reactive ion etching with $\mathrm{SF}_{6} / \mathrm{Ar}$. A micrograph of a device coupling three $\lambda / 4$ resonators to a common feed line is shown in figure $5(\mathrm{a})$. The devices are glued on a printed circuit board, bonded with aluminum bond wires and subsequently characterized in a dilution refrigerator. On the signal input side the experimental wiring of superconducting UTF85 semirigid NbTi cables includes attenuators as thermal anchoring at every temperature stage. The input signal is attenuated by approximately $53 \mathrm{~dB}$, transmitted through the device under test and then amplified by $29 \mathrm{~dB}$ by a low noise HEMT amplifier [35] at the $1 \mathrm{~K}$ stage.

Near its fundamental resonance frequency, given by the length of the resonator $l$ and an effective permittivity $\varepsilon_{\text {eff }}$

$$
f_{\mathrm{r}}=\frac{1}{\sqrt{\varepsilon_{\mathrm{eff}}}} \frac{c}{4 l},
$$

each of the three resonators behaves like a parallel lumpedelement RLC circuit, coupling energy out of the feed line and leading to a distinct resonant drop in feedline transmission $S_{21}$. In the vicinity of the resonance, the transmitted signal can be expressed by [36]

$$
S_{21}=1-\frac{\frac{Q_{1}}{\left|Q_{\mathrm{e}}\right|} \cdot \mathrm{e}^{\mathrm{i} \Theta}}{1+2 \mathrm{i} Q_{1} \cdot \frac{f-f_{\mathrm{r}}}{f_{\mathrm{r}}}},
$$

where $1 / Q_{1}=1 / Q_{\mathrm{i}}+1 / Q_{\mathrm{c}}$. Here, $Q_{\mathrm{i}}$ is the material and temperature dependent internal quality factor of the $\lambda / 4$ structure, and $Q_{\mathrm{c}}$ the geometry dependent coupling quality factor. $Q_{\mathrm{e}}$ is a complex-valued parameter closely related to the coupling quality factor $Q_{\mathrm{c}}$, whose finite phase $\Theta$ can give rise to a line shape asymmetry due to non-ideal circuit elements, e.g. a complex loading of the resonator or impedance mismatches. Its real part fulfils the condition $\operatorname{Re}\left[Q_{\mathrm{e}}^{-1}\right]=Q_{\mathrm{c}}^{-1}$. Figure 5(b) shows the normalized data of the transmission $\left|S_{21}\right|^{2}$ for one exemplary resonance of a pristine film at $T=100 \mathrm{mK}$. It includes a fitted curve following equation (2); an intrinsic quality factor of $Q_{\mathrm{i}} \simeq 23600$ and a coupling quality factor of $Q_{\mathrm{c}} \simeq 10800$ are obtained. Figure 5(c) displays an overview plot of the uncalibrated transmission of a similar device, this time after an exposure of $30 \mathrm{~min}$ to the hot CVD gas mixture. Still, all three resonances can be clearly recognized, with quality factors up to $Q_{\mathrm{i}} \simeq 5000$.

\section{Coplanar resonator temperature dependence}

Figure 6 shows the observed temperature dependence of the resonance frequency $f_{\mathrm{r}}(T)$ (figures 6(a) and (c)) and the internal quality factor $Q_{\mathrm{i}}(T)$ (figures $6(\mathrm{~b})$ and (d)) for two devices. The device of figures 6(a) and (b) (device 1) has been characterized after thin film deposition and patterning, the device of figures 6(c) and (d) (device 2) has been additionally exposed to the nanotube CVD growth environment.

As can be seen in the figure, the resonance frequency $f_{\mathrm{r}}$ clearly decreases at high temperatures $(T \gtrsim 0.8 \mathrm{~K}$ for device $1, T \gtrsim 0.4 \mathrm{~K}$ for device 2 ). This can be attributed to a decrease in superfluid density of the superconducting thin film. A related rise in the quasiparticle density leads to a higher damping of the resonator and therefore to a reduction of the internal quality factor. Following Mattis and Bardeen $[34,37]$, the temperature dependence of $f_{\mathrm{r}}$ can be expressed 

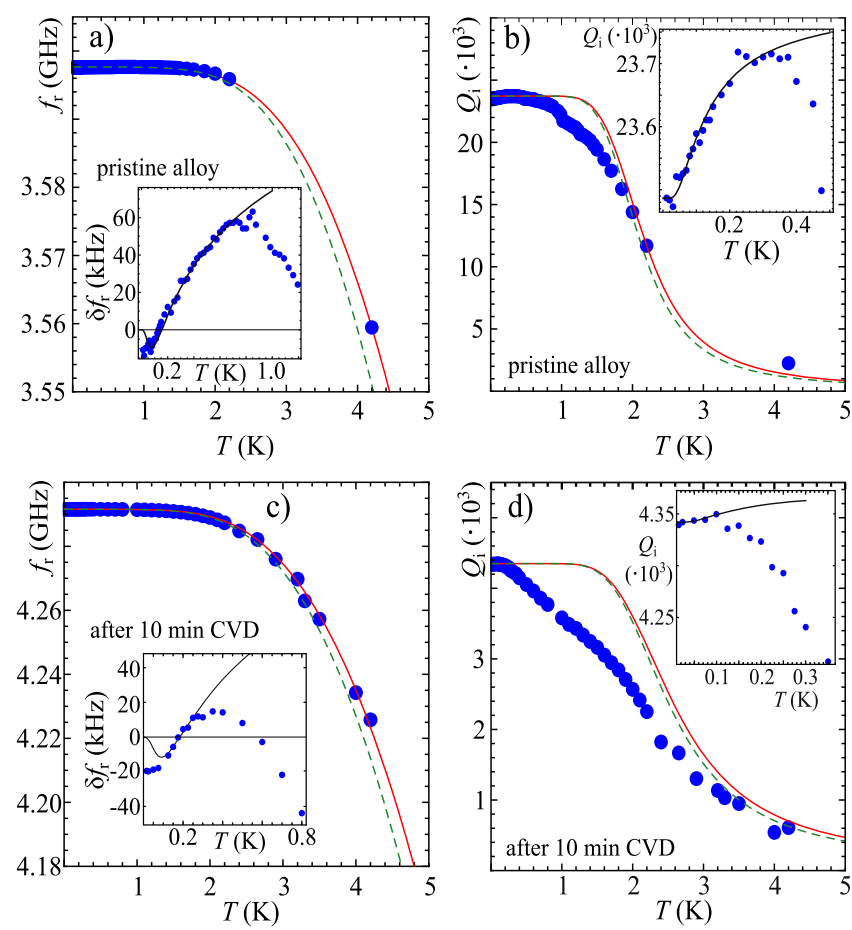

Figure 6. Temperature dependence of resonance frequency $f_{\mathrm{r}}$ and internal quality factor $Q_{\mathrm{i}}$ for two $\lambda / 4$ resonator devices. (a), (b) Resonance frequency $f_{\mathrm{r}}$ (a) and internal quality factor $Q_{\mathrm{i}}$ (b) for device 1 (no CVD treatment); compensation-doped silicon substrate covered by $500 \mathrm{~nm}$ thermal $\mathrm{SiO}_{2}, 10 \mathrm{~nm}$ ALD-deposited $\mathrm{Al}_{2} \mathrm{O}_{3}$ and a $150 \mathrm{~nm}$ thick $\mathrm{Mo}_{20} \mathrm{Re}_{80}$ film. The insets display detail zooms for low temperatures. (c), (d) Corresponding plots for device 2, after undergoing 10 min $\mathrm{CH}_{4} / \mathrm{H}_{2}$ flow in the CVD growth oven.

Compensation-doped silicon substrate covered by $500 \mathrm{~nm}$ thermal $\mathrm{SiO}_{2}$ and $150 \mathrm{~nm} \mathrm{Mo}{ }_{20} \mathrm{Re}_{80}$. For a description of the fit models (solid and dashed lines) see the text.

as

$$
\frac{\delta f_{\mathrm{r}}}{f_{0}}=\frac{\alpha_{0}}{2} \frac{\delta \sigma_{2}}{\sigma_{2}}
$$

with $\delta f_{\mathrm{r}}(T)=f_{\mathrm{r}}(T)-f_{0}$ the deviation of the resonance frequency with finite temperature and $f_{0}=f_{\mathrm{r}}(T=0)$ the approximated resonance frequency at $T=0$. In the limit $h f \ll \Delta(T=0)$ and $k_{\mathrm{B}} T \ll \Delta(T=0)$ the imaginary part $\sigma_{2}$ of the complex conductivity $\sigma$ of the device can be approximated using the temperature-dependent BCS energy gap $\Delta$ and the normal state conductivity $\sigma_{n}$ as [38]

$$
\frac{\sigma_{2}}{\sigma_{n}}=\frac{\pi \Delta}{h f}\left[1-2 \mathrm{e}^{-\Delta / k_{\mathrm{B}} T} \mathrm{e}^{-h f / 2 k_{\mathrm{B}} T} I_{0}\left(\frac{h f}{2 k_{\mathrm{B}} T}\right)\right],
$$

where $I_{0}(x)$ is a modified Bessel function of the first kind. The parameter $\alpha_{0}$ in equation (3) is the kinetic inductance fraction in zero-temperature limit of the coplanar waveguide.

The solid red lines in figures 6(a) and (c) are fit curves corresponding to this model, using in each case $\alpha_{0}$ as a free parameter. The result agrees very well with the experimental data. For device 1 (no CVD) we obtain $\alpha_{0}=0.199$, for device 2 (after $10 \min$ CVD) $\alpha_{0}=0.249$. The value of $\alpha_{0}$ can be calculated from the normal-state conductance $\sigma_{n}$ and the critical temperature $T_{\mathrm{c}}$ following [39]. Using the parameters of our devices, we obtain $\alpha_{\text {th }}=0.243$ and $\alpha_{\text {th }}=0.284$, in reasonable agreement with the fit results. The corresponding functional dependence of $f_{\mathrm{r}}(T)$ is plotted in figures 6(a) and (c) each as a green dashed line.

In figures 6(b) and (d), clearly also the internal quality factor $Q_{\mathrm{i}}$ of the devices decreases at high temperature. Following Mattis and Bardeen, the corresponding change of the quality factor is

$$
\delta\left(\frac{1}{Q_{\mathrm{i}}}\right)=\alpha_{0} \frac{\delta \sigma_{1}}{\sigma_{2}}
$$

with the real part of the complex conductivity [38]

$$
\frac{\sigma_{1}}{\sigma_{n}}=\frac{4 \Delta}{h f} \mathrm{e}^{-\Delta / k_{\mathrm{B}} T} \sinh \left(\frac{h f}{2 k_{\mathrm{B}} T}\right) K_{0}\left(\frac{h f}{2 k_{\mathrm{B}} T}\right) .
$$

Here $K_{0}(x)$ is a modified Bessel function of the second kind. Using the parameters extracted in figures 6(a) and (c) we can plot the corresponding expected temperature dependence $Q_{\mathrm{i}}(T)$, again as solid red and dashed green lines; while significant deviations exist, the overall tendency agrees well with the data.

For $T \ll T_{\mathrm{c}}$, the theory by Mattis and Bardeen predicts no change in the resonance frequency with temperature. However, in our devices for $T \lesssim 0.5 \mathrm{~K}$ a slight decrease in $f_{\mathrm{r}}$ is observed, leading to an overall nonmonotonic behavior of $f_{\mathrm{r}}(T)$, see the insets of figures 6(a) and (c). This is consistent with the influence of two-level systems (TLS) in the substrate contributing to both dissipation and dispersion and can be described by [40-42]

$$
\frac{\delta f_{\mathrm{r}}}{f_{0}}=\frac{F}{2} \frac{\delta \varepsilon}{\varepsilon}
$$

$$
=\frac{F \vartheta}{\pi}\left[\operatorname{Re} \Psi\left(\frac{1}{2}+\frac{1}{2 \pi \mathrm{i}} \frac{h f_{\mathrm{r}}(T)}{k_{\mathrm{B}} T}\right)-\ln \left(\frac{1}{2 \pi} \frac{h f_{\mathrm{r}}(T)}{k_{\mathrm{B}} T}\right)\right] .
$$

Here, $\Psi$ is the digamma function, $0<F<1$ the filling factor, giving the ratio of the electric energy stored in the TLS hosting material to the total electric energy stored in the resonator, and $\vartheta$ the loss tangent of the substrate. The insets of figures 6(a) and (c) show the measured data along with a lowtemperature fit, using the product $F \vartheta$ and $f_{0}$ as fit parameters.

In the case of device 1 , figure $6(\mathrm{a})$, this results in $F \vartheta=3.968 \cdot 10^{-5}$. This value of $F \vartheta$ is comparable to literature values for niobium resonators on sapphire, see e.g. [43]. The fit value for $F \vartheta$ provides an approximation for the TLS-related low-power, low-temperature internal Q-factor $Q_{\mathrm{i}, \mathrm{TLS}} \approx 1 /(F \vartheta) \approx 25000$. As expected this slightly exceeds our measured value of $Q_{\mathrm{i}}(T=20 \mathrm{mK}) \approx 23500$. The fit for device 2 provides $F \vartheta=4.087 \cdot 10^{-5}$. This results in a quality factor $Q_{\mathrm{i}, \mathrm{TLS}} \approx 24500$, comparable to the value for device 1 and apparently insensitive to the degradation of the device due to the CVD process.

Similar to $f_{0}$, also the low-temperature behavior of the quality factor $Q_{\mathrm{i}}$ is governed by interactions with substrate 
TLS. This can be modeled by $[41,42]$

$$
\frac{1}{Q_{\mathrm{i}}}=F \vartheta_{\text {eff }} \tanh \left(\frac{h f_{\mathrm{r}}(T)}{2 k_{\mathrm{B}} T}\right)+\frac{1}{Q_{\text {other }}} .
$$

Here, $\vartheta_{\text {eff }}$ is an effective, reduced loss tangent which takes into account that at strong driving the two-level systems are partially saturated and thereby unable to absorb energy. $Q_{\text {other }}$ describes dissipative processes unrelated to TLS. The fit for device 1 again agrees well with the measured data (inset of figure $6(\mathrm{~b}))$ and results in $F \vartheta_{\text {eff }}=5.677 \cdot 10^{-7}$ and $Q_{\text {other }}=23800$. For device 2 (inset of figure 6(d)) only few data points at low temperature are available, and a saturation of the device temperature cannot be excluded. Applying the fit, we obtain $F \vartheta_{\text {eff }}=1.634 \cdot 10^{-6}$ and $Q_{\text {other }}=4370$, dominating the TLS contribution.

As can be seen in figure 6, the combined effect of twolevel systems and a temperature dependent superfluid density provide a good description of our devices. Due to the CVD process the kinetic inductance fraction increases. The low temperature fits tentatively display similar influence of twolevel systems before and after CVD, however a significant non-TLS induced dissipation term results in the second, postCVD device.

\section{Summary and conclusions}

The XPS characterization demonstrates that our co-sputtering process from two independent targets can generate MoRe thin films of controlled alloy composition. We observe traces of molybdenum oxides at the film surface after ambient air exposure. Exposing the thin films to the carbon nanotube CVD growth environment, a significant amount of carbon is incorporated into the film. No clear indications for the formation of molybdenum carbide can be found; the surface oxide is absent after CVD, pointing towards its reduction in the $850{ }^{\circ} \mathrm{C}_{2}$ atmosphere.

In electrical characterization, we observe a higher resilience to the CVD process for $\mathrm{Mo}_{20} \mathrm{Re}_{80}$ films; the critical temperature only drops below $T_{\mathrm{c}}=8 \mathrm{~K}$ for growth times $>20 \mathrm{~min}$. For shorter growth times, data indicates that the CVD process may have effects similar to the annealing discussed in [32], i.e., enhancing the critical temperature and critical current density. We observe up to $j_{\mathrm{c}} \simeq 2.7 \cdot 10^{5} \mathrm{~A} \mathrm{~mm}^{-2}$ and $T_{\mathrm{c}} \simeq 9.2 \mathrm{~K}$ in a Hall bar geometry.

$\lambda / 4$ coplanar waveguide resonators were defined by thin film deposition and subsequent reactive ion etching. After structuring, we observe internal quality factors up to $Q_{\mathrm{i}}=23700$ in pristine devices and up to $Q_{\mathrm{i}}=5000$ after undergoing the CVD process. The temperature evolution of the resonance frequency $f_{\mathrm{r}}$ and the internal quality factor $Q_{\mathrm{i}}$ of the devices can be understood for low temperatures $T \lesssim 0.4 \mathrm{~K}$ via interaction with substrate two-level systems, at higher temperatures $T \gtrsim 0.8 \mathrm{~K}$ via the decreasing superfluid density.

$\mathrm{Mo}_{20} \mathrm{Re}_{80}$ clearly excels in terms of stability during the CVD process, critical temperature and field as well as critical current. However, the achieved quality factors, also at short exposure to the CVD process, are clearly lower than those reported in literature for similar materials [34]. Further work may be required to precisely determine the origin of the damping, however, our reference niobium devices characterized for comparison have reached $Q_{\mathrm{i}} \simeq 4 \cdot 10^{5}$, excluding the detection setup as cause. The effect of the CVD process in lowering $Q_{\mathrm{i}}$ is similar to other published observations [34]. Future improvements thus should be targeted at the radiofrequency properties of the substrate as well as the the pristine, as-deposited metal film and coplanar waveguide resonators defined in it.

\section{Acknowledgments}

The authors gratefully acknowledge funding by the Deutsche Forschungsgemeinschaft via SFB 631, GRK 1570, and Emmy Noether project Hu 1808/1. We thank F Deppe and R Gross for insightful discussions and experimental advice.

\section{References}

[1] Iijima S and Ichihashi T 1993 Single-shell carbon nanotubes of $1 \mathrm{~nm}$ diameter Nature 363 603-5

[2] Bethune D S, Klang C H, de Vries M S, Gorman G, Savoy R, Vazquez J and Beyers R 1993 Cobalt-catalysed growth of carbon nanotubes with single-atomic-layer walls Nature $\mathbf{3 6 3}$ 605-7

[3] Avouris P 2002 Carbon nanotube electronics Chem. Phys. 281 429-45

[4] Avouris P, Chen Z and Perebeinos V 2007 Carbon-based electronics Nat. Nanotechnol. 2 605-15

[5] Shulaker M M, Hills G, Patil N, Wei H, Chen H-Y, Wong H-S P and Mitra S 2013 Carbon nanotube computer Nature 501 526-30

[6] Grove-Rasmussen K, Jørgensen H I and Lindelof P E 2007 Fabry-Perot interference, Kondo effect and Coulomb blockade in carbon nanotubes Physica E 40 92-8

[7] Steele G A, Götz G and Kouwenhoven L P 2009 Tunable fewelectron double quantum dots and Klein tunnelling in ultraclean carbon nanotubes Nat. Nanotechnol. 4 363-7

[8] Benyamini A, Hamo A, Viola Kusminskiy S, von Oppen F and Ilani S 2014 Real-space tailoring of the electron-phonon coupling in ultraclean nanotube mechanical resonators Nat. Phys. 10 151-6

[9] Schmid D R, Smirnov S, Margańska M, Dirnaichner A, Stiller P L, Grifoni M, Hüttel A K and Strunk Ch 2015 Broken SU(4) symmetry in a Kondo-correlated carbon nanotube Phys. Rev. B 91155435

[10] Gaass M, Hüttel A K, Kang K, Weymann I, von Delft J and Strunk Ch 2011 Universality of the Kondo effect in quantum dots with ferromagnetic leads Phys. Rev. Lett. 107176808

[11] Cao J, Wang Q and Dai H 2005 Electron transport in very clean, as-grown suspended carbon nanotubes Nat. Mater. 4 745-9

[12] Hüttel A K, Steele G A, Witkamp B, Poot M,

Kouwenhoven L P and van der Zant H S J 2009 Carbon nanotubes as ultrahigh quality factor mechanical resonators Nano Lett. 9 2547-52

[13] Moser J, Eichler A, Güttinger J, Dykman M I and Bachtold A 2014 Nanotube mechanical resonators with quality factors of up to 5 million Nat. Nanotechnol. 9 1007-11 
[14] Delbecq M R, Schmitt V, Parmentier F D, Roch N, Viennot J J, Fève G, Huard B, Mora C, Cottet A and Kontos T 2011 Coupling a quantum dot, fermionic leads, and a microwave cavity on a chip Phys. Rev. Lett. 107256804

[15] Viennot J J, Delbecq M R, Dartiailh M C, Cottet A and Kontos T 2014 Out-of-equilibrium charge dynamics in a hybrid circuit quantum electrodynamics architecture Phys. Rev. B 89165404

[16] Ranjan V, Puebla-Hellmann G, Jung M, Hasler T, Nunnenkamp A, Muoth M, Hierold C, Wallraff A and Schonenberger C 2015 Clean carbon nanotubes coupled to superconducting impedance-matching circuits Nat. Commun. 67165

[17] Kong J, Soh H T, Cassell A M, Quate C F and Dai H 1998 Synthesis of individual single-walled carbon nanotubes on patterned silicon wafers Nature 395878

[18] Wu C C, Liu C H and Zhong Z 2010 One-step direct transfer of pristine single-walled carbon nanotubes for functional nanoelectronics Nano Lett. 10 1032-6

[19] Pei F, Laird E A, Steele G A and Kouwenhoven L P 2012 Valley-spin blockade and spin resonance in carbon nanotubes Nat. Nanotechnol. 7 630-4

[20] Testardi L R, Hauser J J and Read M H 1971 Enhanced superconducting $T_{\mathrm{c}}$ and structural transformation in Mo-Re alloys Solid State Commun. 9 1829-31

[21] Gavaler J R, Janocko M A and Jones C K 1972 A-15 structure Mo-Re superconductor Appl. Phys. Lett. 21 179-80

[22] Postnikov V S, Postnikov V V and Zheleznyi V S 1977 Superconductivity in Mo-Re system alloy films produced by electron beam evaporation in high vacuum Phys. Status Solidi A 39 21-3

[23] Schneider B H, Etaki S, van der Zant H S J and Steele G A 2012 Coupling carbon nanotube mechanics to a superconducting circuit Sci. Rep. 2599

[24] Stiller P L, Kugler S, Schmid D R, Strunk Ch and Hüttel A K 2013 Negative Frequency tuning of a carbon nanotube nanoelectromechanical resonator under tension Phys. Status Solidi B 250 2518-22

[25] Talvacchio J, Janocko M A and Greggi J 1986 Properties of evaporated Mo-Re thin-film superconductors J. Low Temp. Phys. 64 395-408

[26] Seleznev V A, Tarkhov M A, Voronov B M, Milostnaya I I, Lyakhno V Y, Garbuz A S, Mikhailov M Y, Zhigalina O M and Gol'tsman G N 2008 Deposition and characterization of few-nanometers-thick superconducting Mo-Re films Supercond. Sci. Technol. 21115006

[27] Moulder J F, Stickle W F, Sobol P E and Bomben K D 1992 Handbook of X-ray Photoelectron Spectroscopy 2nd edn (Eden Prairie, MN: Perkin-Elmer)

[28] Scanlon D O, Watson G W, Payne D J, Atkinson G R, Egdell R G and Law D S L 2010 Theoretical and experimental study of the electronic structures of $\mathrm{MoO}_{3}$ and $\mathrm{MoO}_{2}$ J. Phys. Chem. C 114 4636-45

[29] Wiberg N, Wiberg E and Hollemann A F 1995 Lehrbuch der Anorganischen Chemie 101st edn (Berlin: de Gruyter and $\mathrm{Co}$ )

[30] Stöcker H and Hartnack C (ed) 2007 Taschenbuch der Physik 5th edn (Frankfurt: Harri Deutsch)

[31] Morton N, James B W, Wostenholm G H, Pomfret D G, Davies M R and Dykins J L 1971 Superconductivity of molybdenum and tungsten carbides J. Less Common. Metals 25 97-106

[32] Aziz M, Hudson D C and Russo S 2014 Molybdenumrhenium superconducting suspended nanostructures Appl. Phys. Lett. 104233102

[33] Tinkham M and McKay G 1996 Introduction to Superconductivity 2nd edn (New York: McGraw-Hill)

[34] Singh V, Schneider B H, Bosman S J, Merkx E P J and Steele G A 2014 Molybdenum-rhenium alloy based high-Q superconducting mircowave resonators Appl. Phys. Lett. 105 222601

[35] Caltech CITCRYO1-12A cryogenic microwave amplifier, http://caltechmicrowave.org/

[36] Khalil M S, Stoutimore M J A, Wellstood F C and Osborn K D 2012 An analysis method for asymmetric resonator transmission applied to superconducting devices $J$. Appl. Phys. 111054510

[37] Mattis D C and Bardeen J 1958 Theory of the anomalous skin effect in normal and superconducting metals Phys. Rev. 111 $412-7$

[38] Gao J, Zmuidzinas J, Vayonakis A, Day P, Mazin B and Leduc H 2008 Equivalence of the effects on the complex conductivity of superconductor due to temperature change and external pair breaking J. Low Temp. Phys. 151 $557-63$

[39] Collin R E 2000 Foundations for Microwave Engineering 2nd edn (Piscataway, NJ: IEEE Press)

[40] Phillips W A 1987 Two-level states in glasses Rep. Prog. Phys. 501657

[41] Bruno A, de Lange G, Asaad S, van der Enden K L, Langford N K and DiCarlo L 2015 Reducing intrinsic loss in superconducting resonators by surface treatment and deep etching of silicon substrates Appl. Phys. Lett. 106 182601

[42] Pappas D P, Vissers M R, Wisbey D S, Kline J S and Gao J 2011 Two-level system loss in superconducting microwave resonators IEEE Trans. Appl. Supercond. 21 871-4

[43] Gao J, Daal M, Vayonakis A, Kumar S, Zmuidzinas J, Sadoulet B, Mazin B A, Day P K and Leduc H G 2008 Experimental evidence for a surface distribution of two-level systems in superconducting lithographed microwave resonators Appl. Phys. Lett. 92152505 